\title{
Towards long-distance quantum communication using trapped ions and frequency qubits
}

Ghadimi, M., Connell, S., Scarabel, J., Shimizu, K., Streed, E., et al.

M. Ghadimi, S. Connell, J. Scarabel, K. Shimizu, E. Streed, M. Lobino, "Towards long-distance quantum communication using trapped ions and frequency qubits," Proc. SPIE 11200, AOS Australian Conference on Optical Fibre Technology (ACOFT) and Australian Conference on Optics, Lasers, and Spectroscopy (ACOLS) 2019, 112001E (30 December 2019); doi: $10.1117 / 12.2540050$ 


\title{
Towards Long-Distance Quantum Communication Using Trapped Ions and Frequency Qubits
}

\author{
M. Ghadimi*a , S. Connell ${ }^{\mathrm{a}}$, J. Scarabel ${ }^{\mathrm{a}}$, K. Shimizu ${ }^{\mathrm{a}}$, E. Streed ${ }^{\mathrm{a}, \mathrm{b}}$ and M. Lobino ${ }^{\mathrm{a}, \mathrm{c}}$, \\ ${ }^{a}$ Centre for Quantum Dynamics, Griffith University, Nathan QLD 4111, Australia; \\ ${ }^{\mathrm{b}}$ Institute for Glycomics, Griffith University, Southport QLD 4215, Australia; \\ 'Queensland Micro- and Nanotechnology Centre, Griffith University, Nathan QLD 4111, Australia;
}

\begin{abstract}
Quantum communication requires long-lived memories and robust communication qubits. Long-lived memories and high fidelity two qubit gates are demonstrated using atomic qubits. Frequency qubits are shown to be robust against noises present in long distance communications. In this project we are working towards using trapped ${ }^{171} \mathrm{Yb}^{+}$ions as memory qubits and frequency encoded photonic qubits as communication qubits to realise long distance quantum communication protocols.
\end{abstract}

Keywords: Quantum Communication, Frequency Qubit, Trapped Ion

\section{INTRODUCTION}

Trapped ions are among the most attractive platforms to realise quantum computing and communication. They are well isolated from the environment and can retain their quantum state for minutes ${ }^{1}$. High fidelity deterministic state preparations (99.9999\%), single qubit gates $(99.93 \%)$, and two qubit gates $(99.3 \%)$ have also been realised with trapped ions ${ }^{1,2}$. For quantum communication applications, spontaneously emitted single photons are used to generate ion-photon entanglement. The quantum state of these photonic qubits is entangled with internal state of the atomic qubit and entanglement swapping operations can then be used to remotely entangle two atoms. Recently ion-photon entanglement was demonstrated through $50 \mathrm{~km}$ of fibre by encoding the quantum states in the polarisation degree of freedom ${ }^{3}$. This scheme is only feasible when the fibre is in a lab and isolated from temperature and pressure noises. In a real communication scenario with telecom fibres the transferred state will be in a mixed state. Unlike polarisation encoding, frequency encoding is essentially insensitive to common noise processes in telecommunication setups. A short distance, proof of principle, remote entanglement experiment using frequency qubits has been demonstrated with ${ }^{171} \mathrm{Yb}^{+}$ions ${ }^{4}$. Hyperfine levels of the ground state ${ }^{171} \mathrm{Yb}^{+}$are magnetic field insensitive and can retain the encoded quantum state for a few seconds ${ }^{5}$. State preparation, manipulation and detection can also be done with high fidelity ${ }^{5}$. The only problem with using ${ }^{171} \mathrm{Yb}^{+}$for long distance communication is that the emitted photons are in ultra-violet range (370nm) and suffers high losses when sent through fibres. To overcome this problem, a frequency conversion Lithium-Niobate waveguide is built in our group to convert the frequency to telecom frequencies. After conversion we will use the existing DWDM and CWDM provisioned leased fibres between Nathan and Gold Coast campuses of Griffith University ( $60 \mathrm{~km}$ apart) to send the photons to a Hong-Ou-Mandel interferometer and a coincidence detection will herald entanglement between the ions. This entangled state then can be used to implement protocols like quantum key distribution or quantum teleportation.

\section{COMMUNICATION PROTOCOL}

Our ion qubit is encoded in the hyperfine states $\mid \mathrm{S}, \mathrm{F}=0, \mathrm{~m}_{\mathrm{F}}=0>$ and $\mid \mathrm{S}, \mathrm{F}=1, \mathrm{~m}_{\mathrm{F}}=0>$ of ${ }^{171} \mathrm{Yb}^{+}$to represent $\mid 0>$ and $\mid 1>$ (Fig. 1). Normal cooling laser is resonant to $\mid \mathrm{S}, \mathrm{F}=1, \mathrm{~m}_{\mathrm{F}}=0>$ and $\mid \mathrm{P}, \mathrm{F}=0, \mathrm{~m}_{\mathrm{F}}=0>$ transition. Using an electro-optic modulator, we create a $2.1 \mathrm{GHz}$ sideband that derivers $\mid \mathrm{S}, \mathrm{F}=1, \mathrm{~m}_{\mathrm{F}}=0>$ and $\mid \mathrm{P}, \mathrm{F}=1, \mathrm{~m}_{\mathrm{F}}=0>$ and from $\left|\mathrm{P}, \mathrm{F}=1, \mathrm{~m}_{\mathrm{F}}=0\right\rangle$ the ion decays to $\mid \mathrm{S}, \mathrm{F}=1, \mathrm{~m}_{\mathrm{F}}=0>(\mid 0>$ state) and does not absorb and emit photons anymore. Experimentally we could achieve this state in $4(1) \mu \mathrm{s}$ (averaged over 2000 trials).

State manipulation is done through applying a microwave pulse to microwave rails of the trapping chip that are $100 \mu \mathrm{m}$ away from the ion. We could achieve a $\pi$ rotation with a microwave pulse with duration less than $1 \mu \mathrm{s}$.

AOS Australian Conference on Optical Fibre Technology (ACOFT) and Australian Conference on Optics, Lasers, and Spectroscopy (ACOLS) 2019, edited by Arnan Mitchell, Halina Rubinsztein-Dunlop, Proc. of SPIE Vol. 11200, 112001E - (C) 2019 SPIE · CCC code: 0277-786X/19/\$21 - doi: 10.1117/12.2540050 
For state detection, normal cooling laser is applied. If the ion is in state $\mid 0>$ no light from the ion will be detected and if it is in state $\mid 1>$ it will absorb and emit photons ( $10000 / \mathrm{s}$ with $4 \%$ collection efficiency). We used $1 \mathrm{~ms}$ integration time to measure the state with fidelity of $99.5 \%$. For readout along non-computational axes, a microwave rotation pulse is applied and then the state is measured.

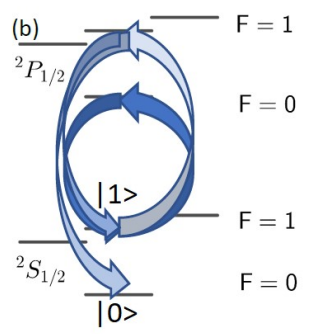

Figure 1. Relevant states of ${ }^{171} \mathrm{Yb}^{+}$.

To generate single photons, we use a microwave pulse resonant to $\mid \mathrm{S}, \mathrm{F}=0, \mathrm{~m}_{\mathrm{F}}=0>$ to $\mid \mathrm{S}, \mathrm{F}=1, \mathrm{~m}_{\mathrm{F}}=-1>$ state. Then we apply a laser pulse resonant to $\mid \mathrm{S}, \mathrm{F}=1, \mathrm{~m}_{\mathrm{F}}=-1>$ to $\mid \mathrm{P}, \mathrm{F}=1, \mathrm{~m}_{\mathrm{F}}=-1>$. From this state the ion will decay to $\mid \mathrm{S}, \mathrm{F}=1, \mathrm{~m}_{\mathrm{F}}=-1>$, $\mid \mathrm{S}, \mathrm{F}=1, \mathrm{~m}_{\mathrm{F}}=0>$ or $\mid \mathrm{S}, \mathrm{F}=0, \mathrm{~m}_{\mathrm{F}}=0>$ with equal probabilities. Photon emitted from decaying to $\mid \mathrm{S}, \mathrm{F}=1, \mathrm{~m}_{\mathrm{F}}=-1>$ can be filtered by a polarizer and the rest of the photons will be in equal superposition of two different frequencies (corresponding to decaying to $\mid \mathrm{S}, \mathrm{F}=1, \mathrm{~m}_{\mathrm{F}}=0>$ or $\mid \mathrm{S}, \mathrm{F}=0, \mathrm{~m}_{\mathrm{F}}=0$ ). The ion also will be entangled with the photon and in equal superposition of $\mid 0>$ and $\mid 1>$. We have already demonstrated the ability to use microwave pulses to drive hyperfine transitions and we have also generated 12ns laser pulses using an electro-optic modulator that we use to excite the ions and generate single photons.

To read the state of the photons we designed an optical setup with a grating with dimensions of $10 \mathrm{~cm}$ by $10 \mathrm{~cm}$ and has 4320 lines per $\mathrm{mm}$. Using this setup, we could sperate the spots corresponding to $12.1 \mathrm{Ghz}$ frequency difference to reach $99 \%$ detection fidelity.

We will continue this project towards realising the long-distance entangling protocol described in the introduction.

\section{ACKNOWLEDGMENT}

This research is financially supported by the Griffith University Research Infrastructure Programme, ML was supported by the Australian Research Council Future Fellowship (FT180100055).

\section{REFERENCES}

[1] Wang, Y., Um, M. Zhang, J., An, S., Lyu, M., Zhang, J.-N., Duan, L.-M., Yum D. and Kim K. "Single-qubit quantum memory exceeding ten-minute coherence time" Nat. Phot. 11, 646-650 (2017).

[2] J. Benhelm, G. Kirchmair, C. F. Roos \& R. Blatt., "Towards fault-tolerant quantum computing with trapped ions" Nature Physics 4, 463-466 (2008).

[3] V. Krutyanskiyy, M. Meranery, J. Schuppy, V. Krcmarsky, H. Hainzer, and B. P. Lanyon, "Light-matter entanglement over $50 \mathrm{~km}$ of optical fibre" arXiv1901.06317v1 (2019).

[4] P. Maunz, S. Olmschenk, D. Hayes, D. N. Matsukevich, L.-M. Duan, and C. Monroe, "Heralded Quantum Gate between Remote Quantum Memories" PRL 102, 250502 (2009).

[5] S. Olmschenk et al., "Manipulation and detection of a trapped $\mathrm{Yb}^{+}$hyperfine qubit" Phys. Rev. A 76, 052314 (2007). 